\title{
High platelet content can increase storage lesion rates following Intercept pathogen inactivation primarily in platelet concentrates prepared by apheresis
}

\author{
H. B. Feys, ${ }^{1,2}$ iD R. Devloo, ${ }^{1}$ B. Sabot, ${ }^{3}$ K. De Pourcq, ${ }^{3}$ J. Coene ${ }^{3}$ \&t V. Compernolle ${ }^{1,2,3}$ \\ ${ }^{1}$ Transfusion Research Center, Belgian Red Cross-Flanders, Ghent, Belgium \\ ${ }^{2}$ Faculty of Medicine and Health Sciences, Ghent University, Ghent, Belgium \\ ${ }^{3}$ Blood Service of the Belgian Red Cross-Flanders, Ghent, Belgium
}

Received: 20 April 2017, revised 25 July 2017, accepted 29 August 2017
Background Pathogen inactivation methods for platelet concentrates are increasingly being used in blood banks worldwide. In vitro studies have demonstrated its effects on storage lesion, but little routine quality control data on blood banking outcomes have been reported.

Materials and Methods Swirling of distributed products was monitored before and after implementation of Intercept pathogen inactivation. Metabolic parameters $\mathrm{pH}$, glucose and lactic acid were determined in a random cohort of expired pathogen-inactivated products. Storage lesion indicators in apheresis concentrates with premature low swirling were compared to concentrates with normal swirling.

Results During validation for implementing Intercept pathogen inactivation, $\mathrm{pH}$ and glucose levels decreased faster in apheresis platelet concentrates with high platelet content than with low platelet content or than in pathogen-inactivated pooled buffy coat-derived products. In routine products, glucose exhaustion was more often found in apheresis compared to buffy coat-derived platelet concentrates despite 3-7\% more plasma carryover in the former. Annual incidence of premature low swirling increased significantly by 50\% following implementation of pathogen inactivation implementation for apheresis but not for pooled buffy coat platelet concentrates. In addition, apheresis concentrates with premature low swirling had a significantly higher median platelet count $\left(5.0 \times 10^{11}\right)$ than unaffected products $\left(3.5 \times 10^{11}\right)$.

Conclusion The risk of increased storage lesion rates following Intercept pathogen inactivation is higher for apheresis than for buffy coat-derived platelet concentrates, especially when platelet contents are higher than $5.0 \times 10^{11}$.

Key words: pathogen inactivation, platelets, quality control

\section{Introduction}

The impact of (Intercept) pathogen inactivation on platelets has been studied extensively in experimental conditions, but quality control data from routine use are less common. Experimental research has demonstrated that

Correspondence: Hendrik B. Feys, Transfusion Research Center, Belgian Red Cross-Flanders, Ottergemsesteenweg 413 B-9000 Gent, Belgium E-mail: hendrik.feys@rodekruis.be quality control parameters [1] of Intercept pathogen-inactivated platelets generally are within predefined ranges [2-4] but with a significant impact on in vitro function [5]. This is caused by irreversible changes in the platelet plasma membrane which affect subsequent intracellular signal transduction [6]. Clinical studies have demonstrated significantly lower corrected count increments at 1 and $24 \mathrm{~h}$, shorter transfusion intervals and increased transfusions of Intercept-treated platelets compared to standard concentrates [7, 8]. However, no differences in

This is an open access article under the terms of the Creative Commons Attribution-NonCommercial License, 
mortality, clinically significant bleeding or severe bleeding were found in a meta-analysis of these trials [9]. Of note, the practical impact on blood component processing is significant because several adaptations in the production chain are required to meet acceptance criteria [10].

Here, we describe the results of our extended QC programme, obtained during the first year of routine pathogen inactivation use. Our quality control routine includes monitoring of $\mathrm{pH}$, glucose and lactic acid on a random subset of expired products as well as monitoring swirling score of every platelet concentrate to be issued. In addition, during the first year of routine pathogen inactivation use, apheresis platelet concentrates with 'premature' low swirling score (i.e. before expiration day) were transferred to the research centre for further analysis and compared to expired day 6 platelet concentrates with normal swirling. This quality control series indicated increased chances of storage lesion following Intercept pathogen inactivation particularly for apheresis platelet concentrates with platelet contents higher than $5.0 \times 10^{11}$.

\section{Materials and methods}

\section{Apheresis platelet concentrate production}

Single-donor platelet concentrates were collected by apheresis using the Trima Accel Automated Blood Collection System (Terumo BCT, Lakewood, CO, USA). Final product volumes and platelet content were set to comply with the Intercept pathogen inactivation method guard bands of 'Large Volume' bag sets (Table 1). Platelet additive solution (SSP+, Macopharma, Tourcoing, France) was added aiming for a fixed plasma carryover of $40 \%(\mathrm{v} / \mathrm{v})$. In case the donor was eligible for double-dose apheresis, volumes above $610 \mathrm{~mL}$ were set, allowing splitting to the compliant volume ranges after collection. These doubledose concentrates were split to two separate 'Large
Volume' bag sets prior to Intercept treatment. 'Dual storage' bag sets were used specifically when a paediatric platelet product $\left(1.6 \times 10^{11}\right.$ platelets $)$ was to be derived from the donation. In that case, splitting took place right after Intercept treatment. All apheresis platelet concentrates were stored in the storage containers of the Intercept illumination set.

\section{Buffy coat-derived platelet concentrate production}

Concentrates prepared by the buffy coat method were from whole blood donations. Six buffy coats were manually pooled as described [5, 11], supplemented with SSP+ additive solution and then treated with Intercept pathogen inactivation prior to storage. In this case, a fixed amount of additive solution of $280 \mathrm{ml}$ was added, resulting in a variable plasma content between 33\% and 37\% (v/v). All buffy coat-derived platelet concentrates were stored in the storage containers of the Intercept illumination set.

\section{Laboratory methods}

Standard quality control included determination of $\mathrm{pH}$, glucose and lactic acid levels of a random cohort of expiring buffy coat and apheresis-derived platelet concentrates.

Prior to distribution, platelet concentrates were routinely assessed for swirling yielding an integral score between 0 and 3 . Products with a low swirling score $\leq 1$ were considered inappropriate for issuing. On the day of assessment, these product numbers were introduced in the electronic tracking system. All products with premature low swirling score were thus counted in the year before (2014) and after (2015) introduction of Intercept pathogen inactivation.

Apheresis platelet concentrates with premature low swirling score were further analysed for platelet count,

Table 1 Projected parameters for platelet concentrates prepared by apheresis

\begin{tabular}{|c|c|c|c|c|}
\hline Yield $\left(\times 10^{11}\right)$ & Collected vol (ml) & Collected conc $\left(\times 10^{3} / \mu \mathrm{l}\right)$ & Final vol (ml) & Final conc $\left(\times 10^{3} / \mu \mathrm{l}\right)$ \\
\hline $3 \cdot 7$ & 128 & 2890 & 193 & 1156 \\
\hline $4 \cdot 2$ & 136 & 3087 & 205 & 1235 \\
\hline $4 \cdot 7$ & 144 & 3264 & 217 & 1306 \\
\hline $5 \cdot 2$ & 152 & 3420 & 229 & 1368 \\
\hline $5 \cdot 7$ & 157 & 3625 & 236 & 1450 \\
\hline $7 \cdot 2$ & 260 & 2769 & 391 & 1108 \\
\hline $8 \cdot 0$ & 260 & 3077 & 390 & 1231 \\
\hline 8.5 & 272 & 3125 & 408 & 1250 \\
\hline
\end{tabular}

$\mathrm{vol}=$ volume; conc $=$ concentration.

All concentrates are automatically supplemented with SSP+ additive solution. 
platelet concentration, mean platelet volume (MPV), $\mathrm{pH}$, lactic acid, glucose and expression of surface receptors by flow cytometry. These laboratory analyses were performed on the day of assessment, thus excluding those flagged during weekends. These concentrates were compared to regularly expiring apheresis products assessed (day 6) with a swirling score $\geq 2$.

Platelet counts and MPV were measured on an automated haematology analyser (pocH-100i; Sysmex, Kobe, Japan). Metabolic parameters were measured on a pointof-care blood gas analyser (Siemens RapidPoint 500, Munich, Germany), but $\mathrm{pH}$ was assessed separately using a calibrated glass electrode at $21^{\circ} \mathrm{C}$. Flow cytometry was performed essentially as described [5]. In brief, expression of P-selectin (anti-CD62P phycoerythrin, BD Biosciences, Erembodegem, Belgium), activated integrin $\alpha_{\mathrm{IIb}} \beta_{3}$ (PAC1 fluorescein, BD Biosciences) and phosphatidylserine (Annexin V Peridinin chlorophyll-Cy5.5; BD Biosciences) was determined using an acoustic focusing flow cytometer (Attune, Life Technologies, Carlsbad, CA, USA). Platelets were incubated with labelled antibodies or ligand for $10 \mathrm{~min}$ at room temperature in $10 \mathrm{~mm} \mathrm{4-(2-}$ hydroxyethyl)-1-piperazineethanesulfonic acid (HEPES) buffer, pH 7.4 with $0.9 \%(\mathrm{w} / \mathrm{v}) \mathrm{NaCl}$ (HBS), $1 \mathrm{~mm} \mathrm{MgSO}_{4}$ and $5 \mathrm{~mm} \mathrm{KCl}$, then diluted a 1000-fold immediately before readout as described before [12]. For Annexin V measurements, buffers were supplemented with $2 \mathrm{~mm}$ $\mathrm{CaCl}_{2}$. For measurements of integrin $\alpha_{\mathrm{IIb}} \beta_{3}$ activation on stimulated platelets, the PAR1 agonist thrombin-related activating hexapeptide SFLLRN (TRAP, Sigma-Aldrich, St Louis, MO, USA) was added at $30 \mu \mathrm{M}$, or cross-linked collagen-related peptide at $5 \mu \mathrm{g} / \mathrm{ml}$ (CRP, University of Cambridge, Cambridge, United Kingdom). The signals of the isotype antibody controls were used to set threshold gates including $0.5 \%$ of 10000 negative events. For annexin $\mathrm{V}$ gating, samples were incubated with annexin $\mathrm{V}$ in the absence of ionized calcium. Median fluorescence intensities and percentage positive events were determined of 10000 cells staining positive for the platelet marker CD61 (anti-CD61 allophycocyanin, Life Technologies).

\section{Statistics}

Sample means were compared by $t$-test or Mann-Whitney for parametric and non-parametric data sets, respectively. Chi-square analysis was used to assess contingency tables. A significance level of 0.05 was used. Computational analysis was with Prism (GraphPad Software Inc, La Jolla, CA, USA).

\section{Results}

Apheresis concentrates with high platelet content show signs of increased storage lesion

Experimental process validation to implement Intercept pathogen inactivation showed that the $\mathrm{pH}$ decreased faster in apheresis concentrates compared to pooled buffy coat platelet concentrates (Fig. 1a). Apheresis units with low platelet content $\left(<1.7 \times 10^{11}\right)$ intended for paediatric use did not show this decline suggesting platelet content plays a role as well. Glucose levels on day 6 were moreover lower in the high content apheresis products (Fig. 1b) compared to pooled buffy coat platelet concentrates, despite 3-7\% more plasma carryover. All concentrates had normal swirling throughout the study. This was confirmed in routine use because the incidence of glucose levels below detection limit was higher in apheresis (5/20) than in buffy coat-derived (1/75) products (Fig. 2). The median (range) platelet content of these products was not different (apheresis $3.7(3.4-5.4) \times 10^{11}$ vs. buffy coat $\left.3 \cdot 9(3 \cdot 0-5 \cdot 4) \times 10^{11}, P=0 \cdot 71\right)$.



Platelet count $\left(10^{11}\right)$



Fig. 1 Metabolic parameters of apheresis and buffy coat-derived platelet concentrates in a validation study to implement Intercept pathogen inactivation. (a) The decrease in $\mathrm{pH}$ over a six day storage period of individual Intercept pathogen-inactivated platelet concentrates prepared by apheresis for paediatric use $\left(<1.6 \times 10^{11}, n=3\right)(\mathrm{X})$, for adult use $\left(>3.0 \times 10^{11}, \mathrm{n}=6\right)(\Theta)$ or prepared by pooling of buffy coats $(n=6)(O)$ is shown in function of the initial platelet count. (b) Glucose concentrations were determined on day 6 of storage for the same individual Intercept pathogen-inactivated platelet concentrates described in panel (a). The dotted horizontal line represents the lower limit (1.1 mm) of detection.

(C) 2017 The Authors.

Vox Sanguinis published by John Wiley \& Sons Ltd on behalf of International Society of Blood Transfusion Vox Sanguinis (2017) 


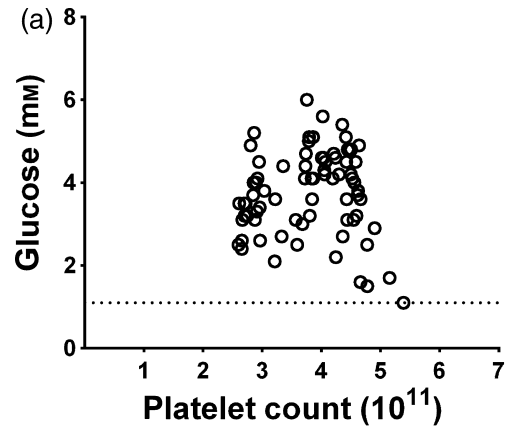



Fig. 2 Glucose concentration in routine platelet concentrates treated with Intercept pathogen inactivation. Glucose concentration was determined in expired (day 6) platelet concentrate units prepared by (a) pooling of buffy coats $(O)$ or by (b) apheresis (O) and is shown in function of the initial platelet count. The dotted horizontal line represents the lowe limit (1.1 mm) of detection for glucose.
Incidence of low swirling was increased in concentrates prepared by apheresis but not by the buffy coat method

In the first year following implementation of Intercept pathogen inactivation, the number of apheresis products with a low swirling score $(\leq 1)$ increased by $50 \%(63 /$ 12 492) compared to the previous year without pathogen inactivation (42/12 931, $P=0.030$, Chi-square). In contrast, the incidence of low swirling score in platelet concentrates prepared by the buffy coat method decreased (2/ 15286 vs. 10/13 488, $P=0.025$, Chi-square) (Fig. 3).

\section{Increased storage lesion in apheresis platelet} concentrates with low swirling score

Next, apheresis platelet concentrates with premature low swirling score $(n=28)$ were compared to regular products



Fig. 3 The number of products with low swirling score before and after introduction of pathogen inactivation. A retrospective analysis of the number of products with a low swirling score $(\leq 1)$ (filled bars) with respect to the total number of distributed products (open bars) was performed on a 12-month record before and after the introduction of Intercept pathogen inactivation. Data are displayed by product type showing apheresis products in black and buffy coat-derived platelet concentrates in blue. expiring on day 5 with normal swirling score $(n=34)$. All were routine products treated with Intercept pathogen inactivation. Apheresis concentrates with low swirling score contained more platelets and at a higher concentration than regular products $(P<0.0001$, Fig. 4a,b). These data sets had a skewed distribution towards the upper range for products with low swirling score as shown in the histograms in Fig. 4(c,d).

Metabolic parameters indicated increased anaerobic metabolism in apheresis concentrates with low swirling because the $\mathrm{pH}$ was decreased $(P<0.0001$, Fig. 5a) and lactic acid concentrations were increased $(P=0.0002$, Fig. 5b) compared to regular products. None of the products had pH levels below 6.4. Glucose concentrations were inversely related to lactic acid (Fig. 5c) and far more products had glucose levels below the detection limit $(1.1 \mathrm{~mm})$ of the blood gas analyser (Fig. $5 \mathrm{~d}$ ) in the cohort with premature low swirling score.

Other storage lesion parameters like MPV (Fig. 6a), annexin $\mathrm{V}$ binding (Fig. 6b) and P-selectin expression (Fig. 6c) were also significantly different $(P<0 \cdot 0001)$. Platelet integrin $\alpha_{\mathrm{IIb}} \beta_{3}$ in products with low swirling score was not prematurely activated (Fig. 6d, $P=0.99$ ) but responded less to TRAP and CRP stimulation (Fig. 6e,f). We moreover found a significant correlation $(P<0.0001)$ between anaerobic respiration and the amount of platelets, especially when combining the data from the premature low swirling products and the regular products (Fig. 7).

\section{Discussion}

The implementation of Intercept pathogen inactivation for platelets demands significant changes in the primary production process of platelet concentrates [10]. To decrease complexity, we chose to only use 'Large Volume' bag sets [13] supplemented with a small number of 'Dual Storage' bag sets for paediatric purposes. Because the Belgian competent authority advocated a minimal platelet dose of $3.0 \times 10^{11}$ for pathogen-inactivated concentrates, our primary apheresis and buffy coat production processes were adapted so to not only meet the Intercept guard bands set 
Fig. 4 Apheresis concentrates with poor swirling contain more platelets than regular products. (a) Platelet count and (b) concentration is shown for apheresis concentrates with abnormally low swirling before expiration $(\leq 1, n=28)$ compared to regular products with acceptable swirling $(\geq 2)$ on the day of expiration $(n=26)$. Data are shown as box and whiskers with median (line) and range (whiskers). (c, d) Histograms show the platelet count and platelet concentration distribution of apheresis concentrates with abnormally low swirling before expiration (filled bars) compared to regular products with acceptable swirling on the day of expiration (open bars). All products were treated with Intercept pathogen inactivation.

Fig. 5 Metabolism of platelet concentrates with low and normal swirling. (a) The $\mathrm{pH}$ of apheresis concentrates with abnormally low swirling before expiration $(\leq 1, n=28)$ compared to regular products with acceptable swirling $(\geq 2)$ on the day of expiration $(n=26)$. Data are shown as box and whiskers with median (line) and range (whiskers). The dashed line shows the lower limit of acceptance for platelet concentrates. (b) The lactic acid and (c) glucose concentration for the same samples, depicted as dot plot with the mean (line) and standard deviation (whiskers). The dashed horizontal line represents the limit of detection (1.1 mM) for glucose concentration determination. (d) The number of products with glucose levels below (filled bars) and above (open bars) detection limit.
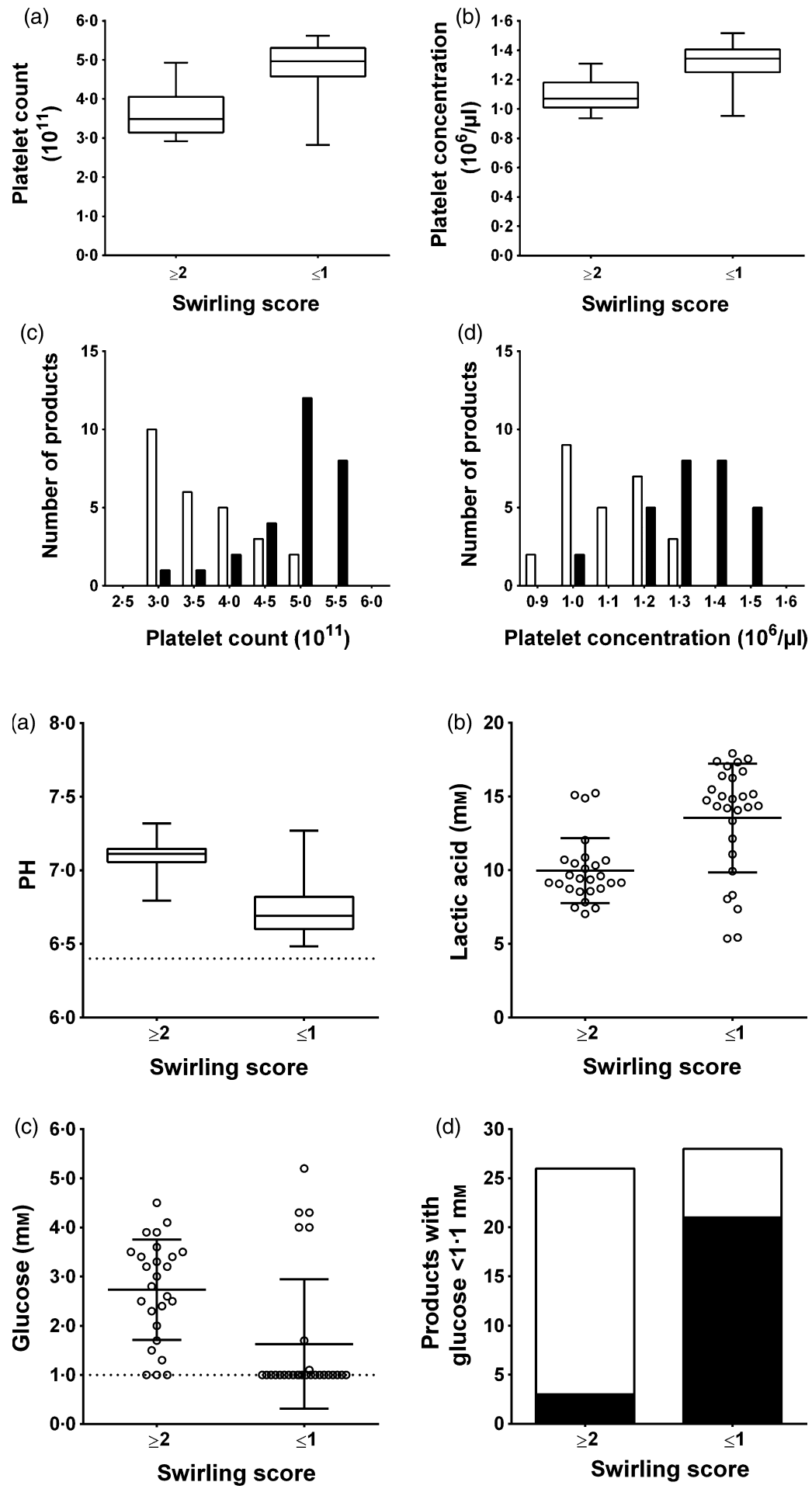

by the manufacturer but also include the critical lower dose limitation. Overall, this has led to platelet concentrates with a relatively high mean platelet content.

The current study shows that platelet content alone does not necessarily relate inversely to quality, at least in the content ranges produced in our facilities and for pooled buffy coat concentrates. Indeed, Intercept pathogen inactivation-treated concentrates prepared from six buffy coats did not show increased incidence of low swirling compared to when pathogen inactivation was not in

\section{(C) 2017 The Authors.}

Vox Sanguinis published by John Wiley \& Sons Ltd on behalf of International Society of Blood Transfusion

Vox Sanguinis (2017) 



Swirling score

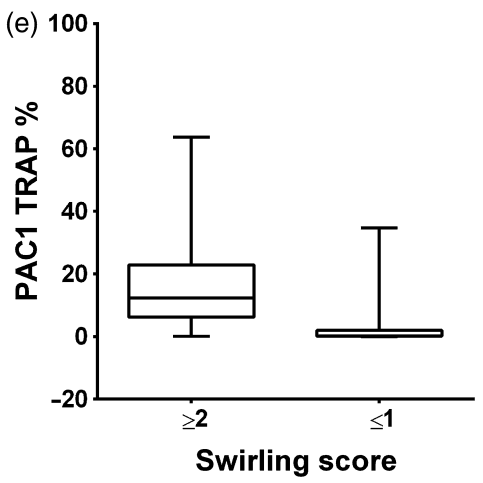



Swirling score

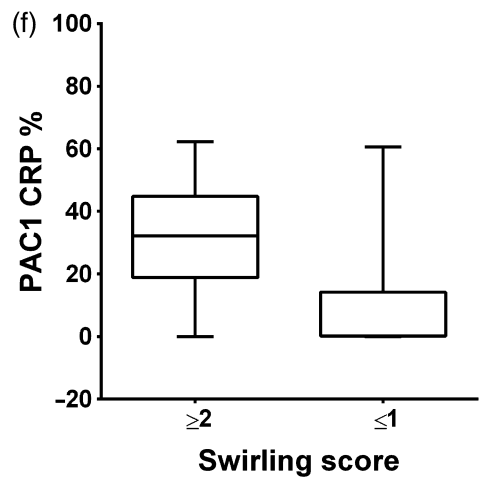

Fig. 6 Storage lesion markers. (a) MPV, (b) annexin $\mathrm{V}$ positive platelets, (c) expression of P-selectin, (d) expression of activated integrin $\alpha_{\| 1} \beta_{3}$ in undisturbed platelets or in platelets activated by (e) TRAP or (f) CRP are depicted as box plots with median (line) and range (whiskers). Apheresis concentrates with abnormally low swirling before expiration $(\leq 1, n=28)$ were compared to regular products with acceptable swirling $(\geq 2)$ on the day of expiration $(n=26)$. Annexin $V_{\text {, }}$ P-selectin and PAC1 data were recorded by flow cytometry and either shown as the percentage positive cells (\%) or the median fluorescence intensity (MFI). place. On the other hand, in the year following implementation of pathogen inactivation, a greater proportion of apheresis platelet concentrates with high platelet content had decreased swirling compared to those prepared from buffy coat, although none of these units had $\mathrm{pH}$ levels below the 6.4 threshold.

The initial validation study for implementation of the Intercept pathogen inactivation process indicated that $\mathrm{pH}$ and glucose levels would decrease faster for apheresis than for buffy coat concentrates despite comparable platelet content, suggesting that apheresis predisposes to increased storage lesion rates. However, because apheresis units with low platelet content $\left(1.6 \times 10^{11}\right)$ did not show this, we reasoned that platelet content must combine with apheresis to generate the effect. In a comparable validation study of Chavarin et al. [14] $(n=16)$, slightly increased storage lesion rates for apheresis products compared to buffy coat-derived products were also found with product quality measures well within standards.

There are several studies that have reported on the quality of apheresis platelet concentrates treated with Intercept pathogen inactivation [3, 14-17]. It is important to note that all of these studies have in common that platelet contents were well below $5.0 \times 10^{11}$ (Table 2) which is relevant because this was the median content of apheresis concentrates that presented with premature low swirling. Furthermore, the published longitudinal research studies require regular sampling in the millilitre range 
Fig. 7 Correlation between platelet count and anaerobic respiration. (a) Lactic acid and (b) $\mathrm{pH}$ were plotted against platelet counts in apheresis concentrates with abnormally low swirling before expiration (O) and regular products with acceptable swirling on the day of expiration (O).The dashed line shows the lower limit of acceptance for platelet concentrates. Spearman's correlation coefficient $(r)$ is shown in the upper left corner of the graph.

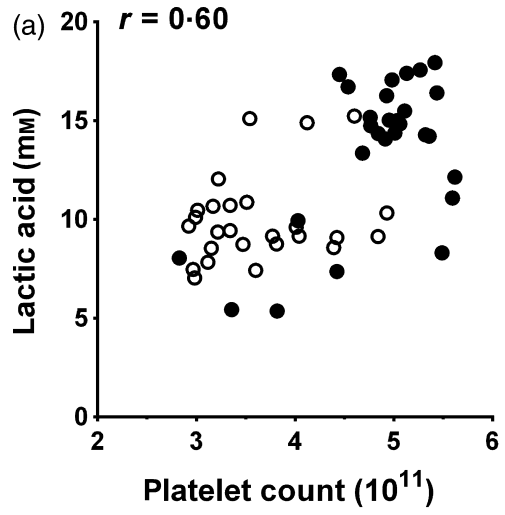

Table 2 In vitro studies on Intercept pathogen-inactivated apheresis platelet concentrates

\begin{tabular}{|c|c|c|c|}
\hline Separator & Content $\left(\times 10^{11}\right)$ & $\mathrm{pH}$ (day 5) & Reference \\
\hline COM.TEC (Fresenius, Bad Homburg, Germany) & $2 \cdot 62 \pm 0.27$ & $6.98 \pm 0.08$ & Moog et al. [15] \\
\hline Trima Accel (Terumo BCT, Lakewood, CO, USA) & $2 \cdot 63 \pm 0.21$ & $6.93 \pm 0.04$ & Picker et al. [16] \\
\hline Amicus (Baxter, Munich, Germany) & $3.62 \pm 0.55$ & $6.99 \pm 0.12$ & Janetzko et al. [3] \\
\hline MCS+ (Haemonetics, Braintree, MA, USA) & $3 \cdot 72 \pm 0.42$ & $6.93 \pm 0.08$ & Isola et al. [17] \\
\hline Amicus & $4.35 \pm 0.91$ & $7 \cdot 08 \pm 0.15$ & Chavarin et al. [14] \\
\hline Trima Accel (Terumo BCT, Lakewood, CO, USA) & $5.08 \pm 0.95$ & $6 \cdot 78 \pm 0 \cdot 15$ & \\
\hline
\end{tabular}

Data are shown as mean \pm SD.

which significantly decreases the actual platelet content during storage thereby potentially biasing the realistic influence of content on storage lesion rates. Overall, the current data set suggests that platelet content is relevant in pathogen-inactivated apheresis concentrates especially above $5.0 \times 10^{11}$.

However, the actual number of apheresis concentrates produced in our blood bank containing more than $5.0 \times 10^{11}$ platelets is estimated to be twenty fivefold higher than the incidence of premature low swirling. This suggests that a noticeable change in swirling indicates end-stage quality loss and cannot be used to monitor ongoing deterioration. Consequently, apheresis concentrates with a high platelet content might have had laboratory signs of increased storage lesion like glucose exhaustion or poor integrin responses but not necessarily poor swirling. This can also be concluded from the initial validation study where platelet concentrates with high content had lowered $\mathrm{pH}$ values and glucose levels at day 5 but normal swirling. Therefore, in the context of Intercept pathogen inactivation, it may be instructive to (re) determine apheresis platelet concentrate quality in function of content especially in ranges above $5.0 \times 10^{11}$ and to investigate whether this influences clinical outcomes like CCI.

Finally, it is difficult to speculate on the reasons why apheresis but not buffy coat-derived concentrates would be more vulnerable in this particular context and for this outcome (low swirling), especially because differences in typical storage lesion markers between buffy coat-derived and apheresis-derived platelet concentrates are minor $[18,19]$. We do think that the actual platelet content in the final (split) apheresis product is of matter and not necessarily the targeted (high dose) one before splitting because double-dose or triple-dose apheresis procedures do not yield products with quickly declining quality, at least when these are split within a couple of hours after donation [20, 21]. In our study, there was no difference between the number of double apheresis donations in the non-swirling group (2/28) versus the normal swirling group (4/26). On the same line, platelet content does not necessarily relate in a linear fashion to $\mathrm{pH}$ or storage lesion [22] in ranges below $5.0 \times 10^{11}[18,23]$. However, only when larger contents are analysed in a scatter plot like in Fig. 7 do such correlations become apparent [24].

In conclusion, real-world quality control data have indicated a small but significant increase in apheresis products with premature low swirling following the introduction of Intercept pathogen inactivation. This was not observed in similarly inactivated products prepared by the buffy coat method. Our data suggest that especially apheresis products with a content above $5.0 \times 10^{11}$ are at risk indicating the need for validation in the upper guard band range for Intercept-treated apheresis concentrates. 


\section{Acknowledgements}

This research was made possible through funding from the Foundation for Scientific Research of the Belgian Red Cross.

\section{Author contributions}

H.B.F., V.C. and J.C. designed research; H.B.F., K.D.P., J.C. and V.C. contributed to critical analytical tools, reagents, samples or data; R.D., B.S. and H.B.F. performed research and collected data; R.D., V.C. and H.B.F. analysed and interpreted data; H.B.F. performed statistical analyses and H.B.F. and V.C. wrote the manuscript; all authors critically reviewed and amended the manuscript.

\section{Conflict of interest}

The authors declare no conflict of interests.

\section{References}

1 Deckmyn H, Feys HB: Assays for quality control of platelets for transfusion. ISBT Sci Ser 2013; 8:221-224

2 Abonnenc M, Sonego G, KaiserGuignard $\mathrm{J}$, et al.: In vitro evaluation of pathogen-inactivated buffy coatderived platelet concentrates during storage: psoralen-based photochemical treatment step-by-step. Blood Transfus 2015; 13:255-264

3 Janetzko K, Lin L, Eichler $\mathrm{H}$, et al.: Implementation of the INTERCEPT Blood System for platelets into routine blood bank manufacturing procedures: evaluation of apheresis platelets. Vox Sang 2004; 86:239-245

4 Apelseth T0, Bruserud 0, Wentzel-Larsen $\mathrm{T}$, et al.: In vitro evaluation of metabolic changes and residual platelet responsiveness in photochemical treated and gamma-irradiated single-donor platelet concentrates during long-term storage. Transfusion 2007; 47:653-665

5 Van Aelst B, Feys HB, Devloo R, et al.: Riboflavin and amotosalen photochemical treatments of platelet concentrates reduce thrombus formation kinetics in vitro. Vox Sang 2015; 108:328-339

6 Van Aelst B, Devloo R, Zachee P, et al.: Psoralen and ultraviolet a light treatment directly affects phosphatidylinositol 3-kinase signal transduction by altering plasma membrane packing. J Biol Chem 2016; 291:24364-24376

7 van Rhenen D, Gulliksson H, Cazenave JP, et al.: Transfusion of pooled buffy coat platelet components prepared with photochemical pathogen inactivation treatment: the euroSPRITE trial. Blood 2003; 101:2426-2433

8 McCullough J, Vesole DH, Benjamin RJ, et al.: Therapeutic efficacy and safety of platelets treated with a photochemical process for pathogen inactivation: the SPRINT Trial. Blood 2004; 104:1534-1541

9 Butler C, Doree C, Estcourt LJ, et al.: Pathogen-reduced platelets for the prevention of bleeding. Cochrane Database Syst Rev 2013; 3:CD009072

10 van der Meer PF, Couture C, Hervig T, et al.: Experiences with semi-routine production of riboflavin and UV-B pathogen-inactivated platelet concentrates in three blood centres. Vox Sang 2017; 112:9-17

11 Hardwick J: Blood processing. ISBT Sci Ser 2008; 3:148-176

12 Goodall AH, Appleby J: Flow-cytometric analysis of platelet-membrane glycoprotein expression and platelet activation. Methods Mol Biol 2004; 272:225-253

13 Irsch J, Lin L: Pathogen inactivation of platelet and plasma blood components for transfusion using the INTERCEPT Blood System. Transfus Med Hemother 2011; 38:19-31

14 Chavarin P, Cognasse F, Argaud C, et al.: In vitro assessment of apheresis and pooled buffy coat platelet components suspended in plasma and SSP+ photochemically treated with amotosalen and UVA for pathogen inactivation (INTERCEPT Blood System ${ }^{\mathrm{TM}}$ ). Vox Sang 2011; 100:247-249

15 Moog R, Frohlich A, Mayaudon V, et al.: In vitro evaluation of COM.TEC apheresis platelet concentrates using a preparation set and pathogen inactivation over a storage period of five days. $J$ Clin Apher 2004; 19:185-191

16 Picker SM, Oustianskaia L, Schneider $\mathrm{V}$, et al.: Functional characteristics of apheresis-derived platelets treated with ultraviolet light combined with either amotosalen-HCl (S-59) or riboflavin (vitamin B2) for pathogen-reduction. Vox Sang 2009; 97:26-33

17 Isola $\mathrm{H}$, Kientz D, Aleil B, et al.: In vitro evaluation of Haemonetics MCS+ apheresis platelet concentrates treated with photochemical pathogen inactivation following plasma volume reduction using the INTERCEPT Preparation Set. Vox Sang 2006; 90:128-130

18 Mitchell SG, Turner VS, Hawker RJ, et al.: A comparative study in volunteers of apheresis and buffy coat derived platelets. Platelets 1995; 6:146-151

19 Krailadsiri P, Seghatchian J: Are all leucodepleted platelet concentrates equivalent? Vox Sang 2000; 78:171-175

20 Macher S, Sipurzynski-Budraß S, Rosskopf $\mathrm{K}$, et al.: Function and activation state of platelets in vitro depend on apheresis modality. Vox Sang 2010; 99:332-340

21 Picker SM, Steisel A, Gathof BS: Cell integrity and mitochondrial function after Mirasol-PRT treatment for pathogen reduction of apheresis-derived platelets: results of a three-arm in vitro study. Transfus Apher Sci 2009; 40:79-85

22 Heaton WA: In-vivo and in-vitro evaluation of stored platelet products; in Lozano M, Sweeney JD (eds): Platelet Transfusion Therapy. Bethesda, MD, AABB Press, 2013:177-227

23 Böck M, Rahrig S, Kunz D, et al.: Platelet concentrates derived from buffy coat and apheresis: biochemical and functional differences. Transfus Med 2002; 12:317-324

24 Wagner SJ, Skripchenko A, Myrup A, et al:: Evaluation of in vitro storage properties of prestorage pooled whole blood-derived platelets suspended in 100 percent plasma and treated with amotosalen and long-wavelength ultraviolet light. Transfusion 2009; 49:704-710 